\title{
WHEN SOAP BUBBLES COLLIDE
}

\author{
COLIN ADAMS AND FRANK MORGAN (WILLIAMS COLLEGE), \\ JOHN M. SULLIVAN (UNIV. ILLINOIS AND TU BERLIN)
}

\section{INTRODUCTION}

Planar soap bubble froths as in Figure 1 have bubbles meeting at most three at a time. Of course, we can create other locally finite decompositions of the plane into closed subsets with disjoint interiors in which arbitrarily many of these pieces meet at a point. But are there decompositions that meet at most two at a point? The answer is yes, as for example occurs with a disk and concentric annuli as in Figure 2. For sets of bounded diameter, however, the answer is no, as we will see shortly.
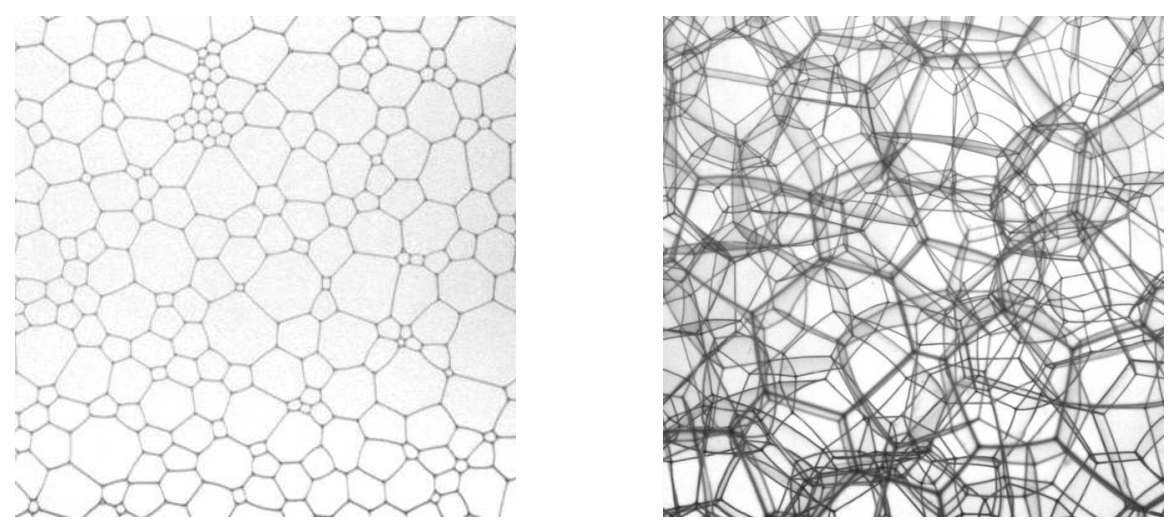

Figure 1. Planar soap bubbles meet in threes. (Photograph, left, taken by Olivier Lorderau at Rennes.) Decompositions of $\mathbb{R}^{3}$ like this soap froth, however, typically meet in fours. (Photograph, right, taken by Sigurdur Thoroddsen at the University of Illinois.)

Similarly, pieces in a decomposition of $\mathbb{R}^{3}$ typically meet in fours, as in Figure 1 Again, we can create examples meeting only in twos, such as a ball and concentric spherical shells. And even when we restrict ourselves to decompositions into pieces that are topologically balls, there are examples whose pieces meet at most in threes. For instance, start with a ball. Cover all but a south polar cap with a pancake. Now cover all but the pancake's north polar cap with a second, southern pancake. Continue layering pancakes, alternating over the top and under the bottom, to fill $\mathbb{R}^{3}$. See Figure 3 for the two-dimensional version, which can be rotated about a north-south axis to obtain the version in three (or higher) dimensions.

Date: 9 August 2004, revised 1 December 2004. 


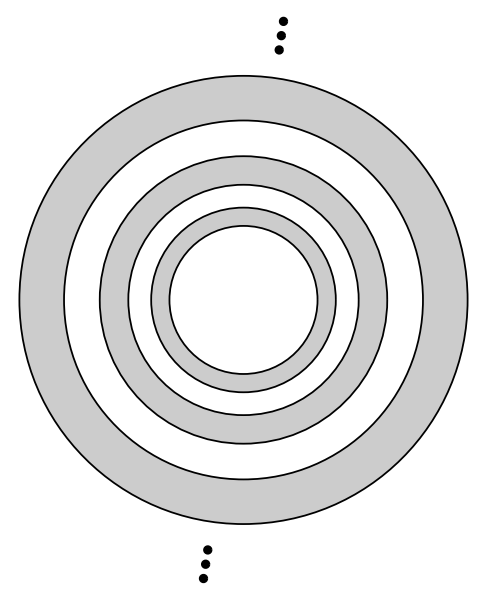

Figure 2. Nested circles decompose the plane into pieces of finite volume (a disk and infinitely many annuli) which meet only in twos. Revolving this figure around a line through the center would give a similar example in three dimensions. This decomposition can be two-colored, as shown.

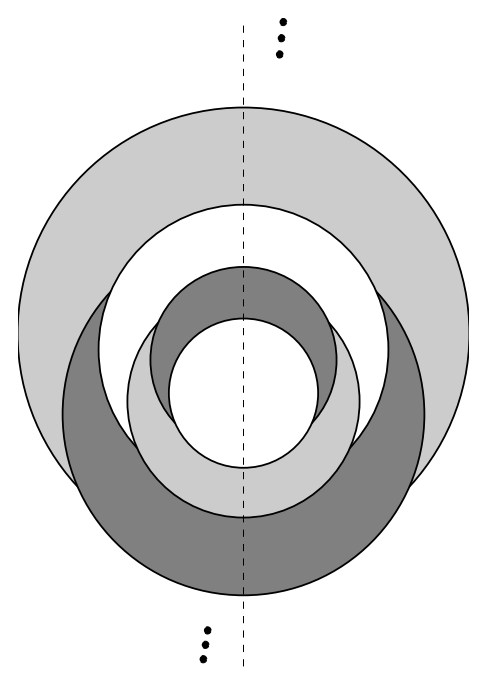

FiguRE 3. This pancake decomposition (of the plane, or of space when revolved around the dashed line) meets only in threes. In fact, it can be globally three-colored, as shown.

If we restrict ourselves to pieces of bounded diameter, however, this cannot occur. In fact, there are no decompositions of any $\mathbb{R}^{n}$ into pieces of bounded diameter meeting at most $n$ at a time. This can be seen from the following version of Lebesgue's Covering Theorem. (Compare [HW] Thm. IV 2].)

Theorem 1.1. The unit n-cube has no decomposition into pieces of diameter less than 1 which meet at most $n$ at a point. 
We will sketch a proof using the Brouwer Fixed Point Theorem in Section 2 Note that this result is sharp, in the sense that the unit $n$-cube does have a piecewise smooth decomposition into sets of diameter at most $1+\epsilon$ which meet at most $n$ at a point. For example, for $n=3$, take the product of an interval with a fine 2-dimensional hexagonal honeycomb in the square; this yields pieces meeting at most three at a time. Similarly for general $n$, take the product of an interval with a fine generic decomposition of the $(n-1)$-cube.

Corollary 1.2. For any Riemannian n-manifold $M$, there is an such that any decomposition into pieces of diameter at most $s$ must have a point where at least $n+1$ pieces intersect.

Proof. A homeomorphic copy of the Euclidean unit $n$-cube $C^{n}$ can always be mapped into $M$ with bounded distortion of distance. Any decomposition of $M$ into pieces with small enough diameters will generate a decomposition of $C^{n}$ into pieces of diameter less than 1 . Hence the pieces of the original decomposition of $M$ must, in places, intersect $n+1$ at a time.

Any decompoisition of $\mathbb{R}^{n}$ into pieces of uniformly bounded diameter could be rescaled to give a decomposition into pieces of diameter less than $s$, so we get as a corollary the result mentioned above.

Corollary 1.3. For $n \geq 1$, there is no decomposition of $\mathbb{R}^{n}$ into sets of bounded diameter meeting at most $n$ at a point.

Similarly, by inscribing an $n$-cube inside the $n$-ball, we also obtain the following.

Corollary 1.4. For $n \geq 1$, there is no decomposition of the unit $n$-ball into sets of diameter less than $2 / \sqrt{n}$ that meet at most $n$ at a point.

We conjecture that this constant $2 / \sqrt{n}$ is not sharp. The best subdivision we know of for the unit $n$-ball is obtained as follows.

We inscribe a regular $n$-simplex in the unit $n$-ball, and then pull the vertices just outside the ball, shaving off that portion of the simplex now outside the ball. The remainder of the simplex will be one of the $n+2$ pieces in our decomposition; its diameter is just over $\sqrt{2+2 / n}$. We divide the exterior of the simplex in the ball into $n+1$ pieces, one for each face of the simplex, by coning the $(n-2)$-skeleton out to the sphere. These pieces have diameter just under $\sqrt{2+\sqrt{2(n-1) / n}}$. Our $n+2$ pieces meet at most $n$ at a point. See Figure 4 for the case $n=3$.

In the case $n=2$, the four pieces in this decomposition have equal diameters $\sqrt{3}$, and in fact we prove below in Proposition 2.4 that this is the minimal-diameter decomposition into pieces meeting at most two at a time. For $n>2$, the truncated simplex can be expanded until its diameter matches the diameters of the other pieces. This appears to yield the minimal-diameter decomposition into pieces meeting at most $n$ at a time.

Implications for soap bubble clusters. Planar soap bubble clusters as in Figure 1are known to meet in at most threes [M1]; triple junctions are always at angles of 120 degrees. Similarly, soap bubble clusters in three dimensions meet in at most fours M1. Corollary 1.4 implies that if the soap bubble cluster covers a ball of diameter at least $\sqrt{3} / 2$ times the largest diameter of a bubble in the cluster, then there must be a point where four bubbles meet. 


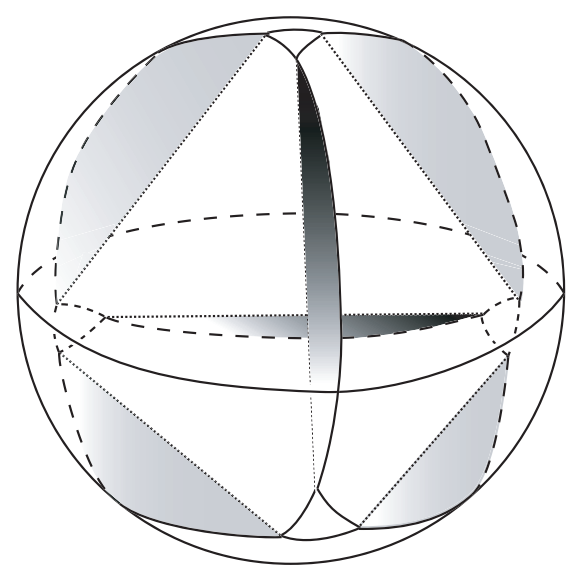

Figure 4. A decomposition of the 3-ball into five regions meeting at most three at a time. To make the decomposition, we use the four faces of a regular tetrahedron-slightly larger than the one inscribed in the ball - together with six "wings" outwards from the edges to the sphere.

Soap bubble clusters in four dimensions will often meet in fives, but another kind of singularity, where bubbles meet in eights - as in the cone over the two-skeleton of the four-cube - is also allowed for soap films Bra (see also [M1]). Hence the results here only give lower bounds on the number of bubbles of bounded diameter that must meet in higher dimensions.

Unfortunately, there are no known useful a priori bounds on the diameter of bubbles in a mimimizing cluster. A minimizing cluster need not be unique, but we can give the following bound on cluster diameter. Actually similar arguments show that the space of such clusters modulo translations is smoothly compact. No such compactness, or even existence, is known for infinite clusters or for variable, unbounded numbers of bubbles.

Proposition 1.5. For any fixed $m$ and $n$, minimizing clusters of $m$ unit volumes in $\mathbb{R}^{n}$ have uniformly bounded diameter.

Sketch of proof. Take any sequence of minimizing clusters. As in [M2, 13.4], using translation we may assume that they converge weakly. (By regularity, a minimizing cluster must be connected, so that no repetitions are necessary to recover all the volume.) By a limit argument ( $\mathrm{Alm}$ or $\mathrm{M} 2,2.1]$ ) the mean curvatures are weakly bounded. By monotonicity ([All, 5.1] or [M1 9.3]) the diameters are bounded.

The Double Bubble Problem in a Riemannian manifold seeks the least-area way to enclose and separate two regions of prescribed volumes. (The complementary outside region then has the complementary volume.) According to a recent theorem of Corneli et al. [C2], in flat two-tori there are five types of solutions, including the

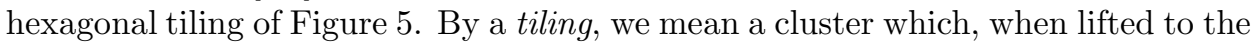
universal cover, gives a partition into regions of finite volume, or equivalently finite diameter. Such tilings provided the main difficulty in [C2]. In the three-torus, Carión et al. C1] conjectured that there are no such minimizing double bubble tilings. That conjecture (and more) follows easily from Lebesgue's Theorem: 


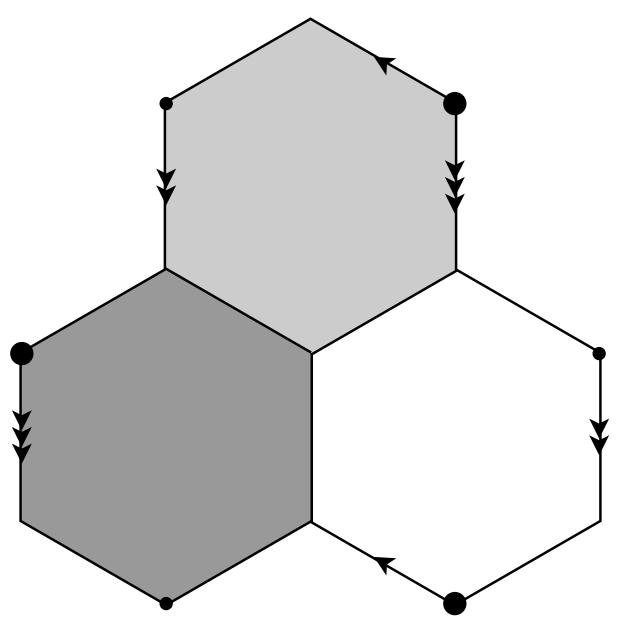

Figure 5. Minimizing double bubbles in flat two-tori include hexagonal tilings $\mathrm{C} 2$, but this can never happen in $n$-tori for $n>2$. Here we see a hexagonal torus divided into three congruent regions. (The torus is obtained from the figure by identifying the boundary as marked; the three vertices marked with small dots get identified, as do the three with large dots.)

Corollary 1.6. In a compact Riemannian n-manifold whose universal cover has infinite injectivity radius, tilings cannot occur as minimizing $m$-bubble clusters for $m \leq n$,

Proof. In the universal cover, such a tiling would decompose a large ball into sets of bounded diameter meeting at most $m$ at a time.

More generally, the Lusternik-Schnirelmann category (see [Fox]) gives a lower bound on the number of contractible components of a decomposition of a manifold. The proof of Corollary [1.6 implies that if the universal cover of a compact $n$ manifold contains large Euclidean balls, then the Lusternik-Schnirelmann Category is $n+1$ (the largest possible value).

Dimension theory. Initially, the concept of the dimension of a space was not carefully delineated, and was inferred fromn the number of coordinates required to describe a point. However, at the end of the nineteenth century, Cantor proved that there was a one-to-one correspondence between the points in a plane and the points on a line. Moreover, Peano proved there was a continuous map of an interval onto a square. It was clear that an appropriate formal definition of dimension was necessary. In 1913, L.E.J. Brouwer introduced a definition of dimension for a topological space, which has evolved into the definition used today. Topologists characterize $n$-dimensional spaces by the property that open covers can be refined to meet at most $n+1$ at all points. (See [HW].) Lebesgue's Theorem 1.1 (actually due to Brouwer $[\mathrm{Bro}]$ ) shows that the topological dimension of $\mathbb{R}^{n}$ is at least $n$. 


\section{ProOfS}

In order to prove Theorem 1.1 we need the following lemma.

Lemma 2.1. Let $\left(F_{i}, F_{i}^{\prime}\right)$ be the $n$ pairs of opposite faces of the unit $n$-cube. Let $B_{1}, \ldots, B_{n}$ be subsets of the cube such that $B_{i}$ separates $F_{i}$ from $F_{i}^{\prime}$. Then $\bigcap_{i} B_{i} \neq$ $\emptyset$.

Proof. Let $C^{n}$ denote the unit $n$-cube. Then $C^{n}-B_{i}$ is the disjoint union of two open sets $U_{i}$ and $U_{i}^{\prime}$ containing $F_{i}$ and $F_{i}^{\prime}$ respectively. For each $x \in C^{n}$, define a vector $v(x)$ whose $i^{\text {th }}$ component is given by $\pm d\left(x, B_{i}\right)$; here we use + if $x \in U_{i}$ and use - if $x \in U_{i}^{\prime}$. Define $f(x):=x+v(x)$. Then $f$ shifts the $i^{\text {th }}$ coordinate of $x$ toward the $i^{\text {th }}$ coordinate of the closest point in $B_{i}$ whose remaining coordinates are the same as those of $x$. Hence, $f$ is a continuous map of $C^{n}$ into itself.

Thus by the Brouwer Fixed Point Theorem, there must be a point $x_{0}$ fixed by $f$. In other words, $v\left(x_{0}\right)=0$ is the trivial vector, so $x_{0}$ lies in the intersection $\bigcap_{i} B_{i}$.

Sketch of proof of Theorem 1.1. Suppose there is a decomposition of the unit cube into pieces of diameter less than 1 meeting at most $n$ at a time, and let $F_{i}$ and $F_{i}^{\prime}$ be the faces of the cube, as above.

Let $L_{1}$ be the union of those decomposition pieces intersecting $F_{1}$. Let $L_{2}$ be the union of those pieces that intersect $F_{2}$ and that were not already used to build $L_{1}$. Continue in this manner to define $L_{3}, \ldots, L_{n}$. Define $L_{n+1}$ to be the union of the remaining pieces.

Define $K_{1}$ to be the boundary of $L_{1}$, given by $L_{1} \cap\left(\bigcup_{i=2}^{n+1} L_{i}\right.$. It separates $F_{1}$ from $F_{1}^{\prime}$. Define $K_{2}$ to be the part of the boundary of $L_{1} \cap L_{2}$ that lies in $K_{1}$. It separates $K_{1} \cap F_{2}$ from $K_{1} \cap F_{2}^{\prime}$. Define $K_{3}, \ldots, K_{n}$ similarly. Note that $K_{n}=\bigcap_{i=1}^{n+1} L_{i}$. By extending the sets $K_{i}$ appropriately, Lemma 2.1 can be used to prove that $K_{n}$ is nonempty. But since no piece was used in the construction of more than one $L_{i}$, this proves that $n+1$ of the pieces from the decomposition intersect.

We next want to derive, in the case $n=2$, the sharp estimate for the diameter of pieces in a partition of the unit $n$-ball meeting at most $n$ at a point. We first quote a standard result from convex geometry, a combination of the Farkas lemma (or separation theorem) and Carathéodory's theorem. (See, for instance, Zie, Prop. 1.10, 1.15].)

Lemma 2.2. Given a finite set $X \subset \mathbb{R}^{n}$, the following conditions are equivalent:

- $X$ lies in no open halfspace bounded by a plane through the origin,

- the origin is in the convex hull of $X$,

- the origin is in the convex hull of at most $n+1$ points in $X$.

Proposition 2.3. Among all configurations of $N$ points on the unit sphere $S^{n-1}$ contained in no open hemisphere, the vertices of the regular $n$-simplex uniquely minimize the maximum distance between points.

Note that the configurations of points that satisfy the hemisphere condition is closed within the space of all configurations, so that a minimizer exists.

Proof. First we claim that for a minimizer, the points lie in no closed hemisphere. If not, $N$ points lie in the bounding great $S^{n-2}$ and in no open hemisphere thereof. By 
induction, some distance is greater than or equal to the edgelength of the inscribed regular $(n-1)$-simplex, which is greater than the edgelength of the inscribed regular $n$-simplex, a contradiction. Thus the hemisphere condition on minimizers is actually open as well as closed. Moreover, any minimizer has at least $n+1$ points. Now suppose that there is some other minimizer. By Lemma 2.2 we may assume that $N=n+1$. From some point the distances to the other points are not all equal. Choose such a minimizer and point $p_{0}$ to minimize the number of other points at maximal distance. We may assume that the distances $d_{1}, \ldots, d_{n}$ from $p_{0}$ to $p_{1}, \ldots, p_{n}$ are nondecreasing and hence that $d_{1}<d_{n}$. Now move $p_{0}$ without changing $d_{2}, \ldots, d_{n}-1$ to decrease $d_{n}$ (and perhaps increase $d_{1}$ ) a bit, contradicting the minimality choice.

Proposition 2.4. An inscribed equilateral triangle provides a least-diameter smooth decomposition of the open unit disc into closed sets which meet at most 2 at a point.

Proof. Suppose that there is such a decomposition into sets of smaller diameter. By replacing each set successively by a small neighborhood we may assume that the decomposition is smooth. By calling each component a set, we may assume that each set is connected. We may assume that the closure of each set touches the unit circle (or absorb the set into the surrounding set), and the boundary of each set is made up alternately of arcs from the circle and chords of the circle. An outermost chord cannot cut off a set that has boundary intersecting the circle in a length greater than $\pi$ since the diameter of this set would be too large. By absorbing outermost regions into adjacent regions of larger diameters, we can assume that the closure of precisely one set $S$ has disconnected intersection with the circle. We may assume that the rest are chordal sections. By Proposition 2.3 $S$ has diameter at least as great as the inscribed equilateral triangle, a contradiction.

Acknowledgements. We would like to thank Joseph Corneli and Stephen Hyde for their inspiring questions and Branko Grünbaum for referring us to Lebesgue's theorem.

\section{REFERENCES}

[All] William K. Allard, On the first variation of a varifold, Ann. Math 95 (1972), 417-491.

[Alm] F. J. Almgren, Jr., Existence and regularity almost everywhere of solutions to elliptic variational problems with constraints, Mem. AMS 165 (1976).

[Bra] Ken Brakke, Minimal cones on hypercubes, J. Geom. Anal. 1 (1991), 329-338.

[Bro] L. E. J. Brouwer, Über den natürlichen Dimensionbegriff, Journ. f. Math. 142 (1913), $146-152$.

[C1] Miguel Carrión Álvarez, Joseph Corneli, Genevieve Walsh, and Shabnam Beheshti, Double bubbles in the three-torus, Exp. Math. 12 (2003), 79-89.

[C2] Joseph Corneli, Paul Holt, George Lee, Nicholas Leger, Eric Schoenfeld, and Benjamin Steinhurst, The double bubble problem on the flat two-torus, Trans. Amer. Math. Soc. 356 (2004), 3769-3820.

[FR] M. Amaral Fortes and M. Emília Rosa, The surface energy and contact angles of a liquid foam, J. Colloid Interface Sci. 241 (2001), 205-214.

[Fox] Ralph H. Fox, On the Lusternik-Schnirelmann category, Ann. Math. 42 (1941), 333-370.

[HW] Witold Hurewicz and Henry Wallman, Dimension Theory, Princeton U. Press (1941).

[M1] Frank Morgan, Geometric Measure Theory: a Beginner's Guide, Academic Press (2000).

[M2] Frank Morgan, Small perimeter-minimizing double bubbles in compact surfaces are standard, Electr. Proc. 78th ann. mtg., La./Miss. Section MAA, Univ. of Miss. (March 23-34, 2001), http://www.mc.edu/campus/users/travis/maa/proceedings/spring2001/

[Zie] Günter M. Ziegler, Lectures on polytopes, GTM 152, Springer (1995). 